Revista de Comunicación y Salud, 2020, Vol. 10, no 3, pp. 53-72

Editado por Cátedra de Comunicación y Salud

ISSN: 2173-1675

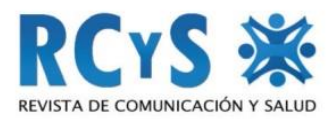

Enviado 25 de febrero de 2020

Aprobado 16 de noviembre de 2020

\title{
USO DE SMARTPHONES EN LA INFANCIA Y SEGUIMIENTO DEL CÓDIGO PAOS POR PARTE DE ANUNCIANTES DE ALIMENTACIÓN
}

\section{Smartphones use in childhood and PAOS code follow-up by food advertisers}

Gloria Jiménez-Marín': Universidad de Sevilla. España.gloria jimenez@us.es

Paloma Sanz-Marcos: Universidad de Sevilla. España. palomasanz@us.es

Rodrigo Elías Zambrano: Universidad de Sevilla. España. rodrigoelias@us.es

Financiación. Este trabajo ha sido realizado en el marco del proyecto $1+D+i$ Retos de Investigación, Representación mediática de la imagen corporal (no)saludable. Desarrollo de una herramienta de prevención en niños y niñas de 5 a 8 años. 'Mi cuerpo me gusta' (CSO2014-58220-R). Dirección General de Investigación Científica y Técnica. Subdirección General de Proyectos de Investigación. Ministerio de Economía y Competitividad.

\section{Resumen}

Enfermedades relacionadas con los hábitos alimentarios, tales como la obesidad, la anorexia o la bulimia, vienen aumentando en sociedades desarrolladas o en vías de desarrollo (Haas et al, 2010). El Ministerio de Sanidad y Consumo del Gobierno de España alertaba, en 2018, de las cifras que alcanzaba la obesidad (15\%) y las EDDs ${ }^{2}$ (12\%): el grupo de edad de mayor incidencia es el de los menores de entre 6 y 12 años (McCabe et al., 2019). Esta cuestión atañe a los media (Levine \& Murnen, 2009; Lara \& Lara, 2018), que se configuran como educomunicadores, debido a la influencia que suponen en los niños al emitir ciertos valores, susceptibles de ser asumidos por estos (Carrillo et al., 2011). En este sentido, la aplicación del "Código de autorregulación de la publicidad y alimentos dirigida a menores, prevención de la obesidad y salud ${ }^{3 \text { " planteaba }}$ un cambio cualitativo y cuantitativo en la publicidad dirigida a niños. El objetivo de esta investigación es analizar la publicidad de alimentos emitida en dispositivos móviles dirigida a menores para comprobar si, efectivamente, este cambio ha sido real. Para ello se utilizó una metodología mixta con una muestra resultado de haber grabado durante 7 días (19 - 25 de agosto de 2019) la publicidad emitida por los anunciantes en el momento

${ }^{1}$ Gloria Jiménez-Marín: Universidad de Sevilla. gloria jimenez@us.es

Lda. en Periodismo, Lda. Publicidad y RR.PP. y Doctora por la Universidad de Sevilla. Profesora Titular en el área de Publicidad y RR.PP. en la Facultad de Comunicación de la misma universidad y docente en la Universitat Oberta de Catalunya.

${ }^{2}$ EDDs: Eating Disorders Diseases

${ }^{3}$ Código PAOS 
Uso de smartphones en la infancia y seguimiento del Código PAOS por parte de anunciantes de alimentación

de interacción con los niños. En concreto: un análisis de contenido de la publicidad objeto de estudio, completada con una encuesta a niños y progenitores, además de un focus group realizado a progenitores.

Palabras clave: Alimentación; educomunicación; medios; niños; publicidad; salud; smartphone.

\begin{abstract}
Diseases related to eating habits, such as obesity, anorexia or bulimia, are on the rise in developed and developing societies (Haas et al., 2010). In 2018, the Spanish Ministry of Health and Consumer Affairs warned about the figures for obesity (15\%) and DIDs (12\%): the age group with the highest incidence is children between 6 and 12 years old (McCabe et al., 2019). This question concerns the averages (Lara \& Lara, 2018; Levine \& Murnen, 2009 ;), which are configured as educators, due to the influence they have on children by emitting certain values, which may be assumed by them (Carrillo et al., 2011). In this sense, the application of the "Code of self-regulation of advertising and food aimed at minors, obesity prevention and health" proposed a qualitative and quantitative change in advertising aimed at children. The aim of this research is to analyse the advertising of food on mobile devices aimed at minors to see if this change has been real. This was done using a mixed methodology with a sample of advertisers' recorded advertising for 7 days (19 - 25 August 2019) when interacting with children. Specifically: a content analysis of the advertisements studied, completed with a survey of children and parents, as well as a focus group with parents.
\end{abstract}

Keywords: Advertising; children; educommunication; food; health; media; smartphone.

\title{
Cómo citar el artículo
}

Jiménez-Marín, G., Sanz-Marcos, P. y Elías Zambrano, R. (2020). Uso de smartphones en la infancia y seguimiento del Código PAOS por parte de anunciantes de alimentación. Revista de Comunicación y Salud, 10(3), 67-86. doi: https://doi.org/10.35669/rcys.2020.10(3).53-72

\section{INTRODUCCIÓN}

Usamos la expresión 'epidemia' para aludir a enfermedades relacionadas con los trastornos alimentarios y los malos hábitos alimenticios, como pueden ser la obesidad, la bulimia o la anorexia nerviosa. El incremento de estas enfermedades es una realidad a nivel internacional. Más aún en el caso de los menores de 12 años, cuyos datos son alarmantes: El Ministerio de Sanidad y Consumo viene aportando datos desde 2006 y la Organización Mundial de la Salud (WHO -World Health Organization-) estima que la obesidad infantil se sitúa sobre el 14\%, con indicadores de que el sobrepeso alcanza cifras superiores al $12 \%$. Así, los datos encontrados en el estudio realizado por JiménezMorales (2006, p. 246) lanzaban cuestiones como que la obesidad infantil se triplicó en España, encontrándose en el segundo lugar de entre los países europeos en la

Revista de Comunicación y Salud, 2020, Vol. 10, n 3, pp. 53-72 
Uso de smartphones en la infancia y seguimiento del Código PAOS por parte de anunciantes de alimentación

prevalencia del exceso de peso entre menores de 6 a 12 años durante ese periodo. No obstante, desde el año 2015 se viene observando una reducción de 3 puntos del sobrepeso en relación con años anteriores, lo que confirmaría cierta estabilización, además de la inversión de la tendencia (Ortega et al., 2015).

En paralelo, destacamos que existe un mercado donde la posibilidad de encontrar una amplia oferta de productos ultraprocesados es muy elevada. Ello viene acrecentado por el hecho de que los receptores y consumidores potenciales son destinatarios de una excesiva cantidad de estímulos publicitarios, muchos de ellos dirigidos a menores, que suponen un colectivo más que sensible y vulnerable (Del Mar Pàmies, Ryan \& Valverde, 2016). Y ello, a través del medio de comunicación móvil por excelencia: el smartphone.

Cierto es que los medios no son los únicos responsables de la epidemia, señalando para ello a la industria alimentaria, claramente. Pero sí suponen un factor importante a considerar. Debido a que la publicidad puede generar representaciones que impliquen patrones de imagen y conductas no saludables (Carlson \& Clarke, 2014; Elías, JiménezMarín \& Silva, 2017), ello puede perturbar directamente la autoestima de los menores y, con ello, la construcción de su propia imagen corporal.

\subsection{Los media y la construcción de la imagen corporal}

La capacidad de influencia que tienen los medios de comunicación, en general, puede advertirse ya desde los estudios realizados a nivel mundial por autores como los de McCabe y Ricciardelli (2005), o los de de Skemp-Arlt et al. (2006), donde se muestra que la mitad de los niños de entre 5 y 12 años se sienten insatisfechos con su aspecto físico. $Y$ es que, tal y como afirman McCabe et al. (2019), Mancilla et al. (2012), Skemp-Arlt et al. (2006), McCabe \& Ricciardelli (2005), Phares et al. (2004), Davison et al. (2003), Hendy et al. (2001) o Abramovitz \& Birch (2000), alrededor de un 40-50\% de los niños entre 6-12 años se sienten insatisfechos con su aspecto físico. De hecho, las evidencias científicas (De Jans et al., 2019) constatan que esta insatisfacción afecta a ambos géneros, pero el tipo de distorsión difiere en función del género. Normalmente, las chicas desean un cuerpo más delgado, mientras que los chicos desean un físico más musculado con un mínimo de grasa corporal. Por tanto, debemos prestar gran atención a estas diferencias de género si queremos desarrollar contenidos específicos sobre imagen corporal (Bird et al., 2013; Frederick et al., 2005).

En este sentido se puede afirmar que los menores son el grupo social más sensible a las consecuencias negativas de los media (Charry, 2014), suponiendo, en gran parte, en dependientes del mercado del consumo (INTEF, 2005). Así, numerosos estudios consideran que la reducción a la exposición de los contenidos emitidos por los medios de comunicación, en especial a la publicidad infantil (Smith et al., 2019; Folkvord et al., 2016; Matthes \& Naderer, 2015), resulta beneficioso para niñas y niños, para su salud física y mental. Es más, gran parte de las investigaciones sobre trastornos en la imagen corporal señalan directamente a los media como productores de estereotipos físicos y, por tanto, entre otros, de la manifestación de desórdenes alimentarios (Botta, 1999; Harrison \& Cantor, 1997; Myers y Biocca, 1992; Stice et al., 1994; Hamilton \& Waller, 1993). Por ello

Revista de Comunicación y Salud, 2020, Vol. 10, no 3, pp. 53-72 
se incide en el hecho de que los menores poseen menos recursos cognitivos para decodificar correctamente los mensajes recibidos por los medios de comunicación (Kapferer, 1995; Unnikrishnan \& Bajpai, 1996; McLean et al., 2013; Fernández \& Díaz, 2014;).

Según la Teoría del Aprendizaje Social, a través de la exposición a contenidos mediáticos vinculados con el atractivo social, la persona (niñas/os y adolescentes en particular) aprehende qué modelos de apariencia física son los socialmente recompensados, las expectativas sociales de lo considerado bello frente a lo considerado feo, y las consecuencias de no ser atractivo. Por su parte, la Teoría del Cultivo (Gerbner, 1998) afirma que la realidad social es moldeada por la exposición extensiva y acumulativa a los mensajes mediáticos. Este modelo teórico asume que los individuos desarrollan creencias, actitudes y expectativas sobre el mundo real en base a lo que ven y escuchan en televisión, vídeo, películas, revistas, etc. Posteriormente, utilizarán estas creencias, actitudes y expectativas para tomar decisiones y adoptar comportamientos en situaciones del mundo real. Así, en el marco de un estudio sobre imagen corporal y contenidos mediáticos, la Teoría del Cultivo plantea que los mensajes mediáticos son agentes de socialización sobre los conceptos de belleza femenina y masculina, y demás aspectos de la imagen corporal. Y esto es particularmente cierto en audiencias infantiles, dado que la programación infantil utiliza mensajes que ponen en juego vínculos emocionales fuertes.

Paralelamente a estos estereotipos, los mismos medios lanzan otro tipo de mensajes contradictorios, promocionando a menudo conductas y productos pocos saludables o con alto contenido en grasas y azúcares durante la programación infantil. Esto es: productos alimentarios ultraprocesados, cultura del premio como recompensa por ingerir una gran cantidad de alimentos, etc. Esta cuestión es altamente sorprendente pues se observan, de hecho, dos secciones completamente diferenciados por las estrategias mediáticas: contenidos que muestran niños felices gracias al consumo de azúcares y grasas en forma de bollería, golosinas o comida rápida, frente a aquellos en los que aparecen adultos felices y delgados a merced, básicamente, del consumo de alimentos bajos en calorías. Esta dicotomía acaba influyendo en el público infantil que, en edades tempranas, rechaza su imagen corporal, iniciando dietas restrictivas que pueden conducirles a trastornos alimentarios (Jiménez-Morales, 2006). En el plano teórico, la directiva 'Televisión Sin Fronteras' de la Unión Europea (89/552/CEE y modificación 97/36/CE) especifica que la "publicidad televisada no deberá perjudicar moral o físicamente a los menores" (art.1) y prevé que los estados miembros vigilen las emisiones televisivas para que no incluyan ningún programa "que pueda perjudicar seriamente el desarrollo físico, mental o moral de los menores" (art. 22). En consecuencia, tanto la programación televisiva como los contenidos publicitarios deberían evitar la representación de conductas de vida y patrones de belleza poco saludables.

\subsection{El Código PAOS}

Revista de Comunicación y Salud, 2020, Vol. 10, n 3, pp. 53-72 
La actual situación ha traído consigo estrategias para prevenir la obesidad infantil y las enfermedades relacionadas con los trastornos alimentarios, así como el fortalecimiento de tácticas que ayuden a incrementar un estilo de vida saludable. Sin embargo, las regulaciones dirigidas a la prevención son bastante limitadas, tanto en Estados Unidos como en Europa, zonas geográficas de referencia en esta cuestión. En ambos territorios existe cierta regulación, pero ella es bastante relajada en relación con el cumplimiento de sus códigos de emisión de imágenes en los medios y de fomento de otras iniciativas de hábitos de vida saludable.

Siguiendo a Sánchez (2016) observamos que, con la finalidad de potenciar hábitos más saludables, se vienen llevando a cabo diferentes estrategias, tales como reducir impuestos a familias participando en actividades deportivas (caso de Canadá) o el uso de gravámenes y cuotas para desincentivar comportamientos no saludables en aquellos empleados que exceden ciertos niveles de perímetro de cintura (caso de Japón). También se han promocionado iniciativas para incrementar la variedad y calidad en el consumo de frutas y verduras (caso de USA o UK) o la regulación del acceso a los alimentos y bebidas con alto contenido en grasa, azúcar o sal en las escuelas y control y regulación de publicidad de alimentos y bebidas por códigos autorreguladores (caso de España) o regulaciones específicas para influir en las decisiones de compra de alimentos y bebidas como etiquetados específicos con los colores de semáforos (UK), etc. además de que muy pocas empresas cumplen dicha regulación correctamente. Con esto se demuestra que la propia autorregulación no es efectiva y que se deberían tomar otras medidas para que los menores estén realmente protegidos frente a las campañas abusivas de los anunciantes como la adopción de un sistema de estatutos que use un modelo de perfil nutricional para restringir la exposición de productos no saludables o imponiendo una prohibición legal a cualquier tipo de anuncio de comida y bebida dirigido a menores.

A nivel europeo, la European Food Safety Authority (EFSA) supone un organismo independiente encargada de asesorar a los distintos gobiernos sobre la existencia de riesgos alimentarios y epidemias o pandemias emergentes. Su objetivo es proteger la seguridad pública con acciones como el impulso de investigaciones científicas, el asesoramiento, la divulgación o el desarrollo de acciones sobre seguridad alimentaria.

En el caso de España, dependiente de la EFSA se cuenta con la atención correguladora de la Agencia Española de Consumo, Seguridad Alimentaria y Nutrición (AECOSAN). Adscrito al Ministerio de Sanidad, Consumo e Igualdad tiene como principal herramienta el Código de Corregulación de la Publicidad de Alimentos y Bebidas Dirigida a Menores, Prevención de la Obesidad y Salud (Código PAOS), enmarcado en la estrategia global estatal por el bienestar social del Gobierno de España, una disposición que afecta al público infantil en el fomento de una vida y dieta saludable.

El Código PAOS se reforzó en el año 2009 mediante un acuerdo de colaboración con los principales operadores de televisión en España de modo que las televisiones se comprometieron a exigir que los anuncios de alimentos y bebidas dirigidos a menores de 12 años (o que se emitiesen en las franjas de protección reforzada de la infancia), 
Uso de smartphones en la infancia y seguimiento del Código PAOS por parte de anunciantes de alimentación

cumplían con las normas establecidas en el Código PAOS. En 2012 el Ministerio de Sanidad, Servicios Sociales e Igualdad y la AECOSAN tuvieron que suscribir un nuevo Código PAOS, donde se sumaron la Federación Española de Industrias de Alimentación y Bebidas (FIAB), la Asociación para la Autorregulación de la Comunicación Comercial (Autocontrol de la publicidad), el sector de la distribución (ANGED, ASEDAS y ACES), la hostelería (FEHR) y la restauración (FEHRCAREM).

Esta actualización del Código PAOS trajo como efecto que ahora el código se aplica también a la publicidad emitida en internet y en dispositivos móviles, que son aquellos donde la audiencia infantil más está creciendo en los últimos años (INFOADEX, 2019). Del mismo modo, la edad de aplicación se extiende hasta los 15 años.

En un estudio realizado entre el 2008 y el 2012 se demostró el incumplimiento del código PAOS aumentando de un $50 \%$ en 2008 a un $88,2 \%$ en 2012 . En la franja de protección reforzada este incumplimiento del código subió de un $43 \%$ a un $86 \%$.

Y es este aspecto el que prevalece en esta investigación: el uso de personalidades conocidas por los menores, personajes famosos o conocidas por estos, en la que más se ha incrementado este incumplimiento subiendo de un 5,4\% a un $25 \%$ (León-Flandez et al., 2012). En una investigación realizada por Hastings et al. en 2003 se pudo ver cómo los anuncios se insertaban dentro de la franja horaria infantil y que utilizaban técnicas publicitarias no convencionales como el patrocinio, reparto de muestras o regalos al comprar productos, como por ejemplo juguetes. Posteriormente, una investigación realizada por la propia Unión Europea demostró que los niños que no veían la televisión en el almuerzo tenían menos probabilidades de tener sobrepeso que los que sí la veían (Vik et al. 2013).

Grupos multimedia también han tomado conciencia de la problemática de la salud de los más pequeños y algunos conglomerados, como A3Media (en el caso de España), realizaron en el año 2012 una campaña llamada 'El Estirón' que se dirigía a menores con obesidad infantil. La campaña estaba compuesta por anuncios en los canales: Antena 3, Neox, Nova y Nitro y en las emisoras de radio Onda Cero y Europa FM. En la campaña participaban algunas personalidades famosas que daban consejos para llevar una vida saludable. A nuestro juicio, al ser una acción aislada y sin un contexto público, político, social y familiar que lo haya reforzado, las posibles consecuencias positivas de esta acción de disolvieron.

\subsection{El uso de dispositivos móviles en menores}

Pueden establecerse ciertas relaciones de conexión directa entre los estereotipos plasmados por la publicidad, de un lado, y la capacidad de esta para influir en la sociedad, por otro. Y, más concretamente, en la capacidad de influir en los menores, que son altamente susceptibles a dejarse persuadir por tales influencias. Como resultado: Una alta insatisfacción de niñas y niños con sus cuerpos. Como consecuencia de este resultado: Trastornos físicos y psicológicos.

Revista de Comunicación y Salud, 2020, Vol. 10, oㅜ 3, pp. 53-72 
Uso de smartphones en la infancia y seguimiento del Código PAOS por parte de anunciantes de alimentación

Hoy disponemos de una amplia variedad de contenidos conectados permanentemente, y de dispositivos capaces de soportarlos (Pérez, 2008). Están accesibles en cualquier lugar y en cualquier momento, a través de distintas plataformas

Hoy en día puede constatarse que entre los menores de quince años el medio más consumido es la televisión, medio convencional, cuyos contenidos se visionan durante su tiempo de ocio.

De hecho, "frente a los nuevos espacios digitales idealizados, amados, envidiados, pero también temidos, está la permanente figura de la televisión, que se mantiene firme en el imaginario colectivo como el espacio familiar de ocio" (Gewerc et al., 2017, p. 178). Sin embargo, inmediatamente después encontramos que el segundo medio más consumido por los menores es internet, fundamentalmente a través de smartphones (INFOADEX, 2019), no detectándose entre este tipo de público el consumo ni de prensa ni de radio convencional (Navarro et. al, 2012). Al tratarse de nativos digitales, internet está presente en su vida cotidiana.

En el caso concreto de los menores de 12 años, la televisión compite, a gran escala, con el smartphone, y no tanto con el ordenador de sobremesa y portátil que, para esta franja de edad, está quedando relegado. A partir de los 12 años lo valores cambian (INFOADEX, 2019). De hecho, el nivel de penetración de los dispositivos móviles a la edad de 9-12 años alcanza un valor relativo del 72,7\%, según datos de AIMC (2017). Este porcentaje aumenta si ampliamos el rango de edad. En este sentido, Bringué \& Sábada (2011) aportan que el terminal móvil alcanza porcentajes del 83\% en niños con edades superiores a los 10 años.

Nos encontramos, pues, ante una generación que se ha adueñado de la cultura comunicativa basada en el uso de las tecnologías digitales y móviles, cada vez más asequibles y universales (Brigué y Sábada, 2011). Se tiene acceso a todo tipo de pantallas con un amplio porcentaje que tiene acceso habitual a Internet.

\section{OBJETIVOS}

En la esfera alimentaria, a nivel internacional, nunca se ha tenido un nivel de seguridad tan alto como el actual y, sin embargo, los problemas de salud relacionados con los alimentos no disminuyen.

En el año 2001 la OMS acuñó por primera vez el término "Globesity" para definir la gravedad de la pandemia que suponía la obesidad en el mundo. En este sentido, ciertos modelos predictivos utilizados por el UK Foresight Report han sugerido que el $55 \%$ de la población británica podrían ser obesos en el Reino Unido en el año 2050. 
Las estimaciones de prevalencia de obesidad y/o sobrepeso en los países $\mathrm{OCDE}^{4}$ y otros emergentes en la población infantil entre 5-17 años aportan valores medios de alrededor de un 22\% (Sánchez, 2016). Uno de cada 5 niños se encuentra enfermo de obesidad en un gran número de países, situándose este valor en uno de cada 3 en países como Grecia, USA o Italia. Por el contrario, el sobrepeso afecta a menos del $10 \%$ en países como China, Corea o Turquía.

Surge, pues, el planteamiento: ¿Es lícito someter a tal cantidad de estímulos a un colectivo, débil e inmaduro como son los menores? ¿Por qué no se trabaja para optimizar de una manera rigurosa ciertos códigos y legislaciones? Ya sea por doctrina capitalista, ya sea por otros motivos adyacentes, las agencias publicitarias y demás sistemas mercadotécnicos, fijan su mercado-meta, su target, en el público infantil, planteando a los menores como prescriptores y consumidores, aunque no necesariamente como compradores. Esta práctica no es mera exaltación de la norma entre principios económicos, sino que es fruto del utilitarismo donde se antepone la alimentación saludable y el factor educacional a la comida envasada, ultra procesada y de fácil uso para el público general. De este modo, la atracción de las marcas de alimentación infantil parte de la psicología conductual de los niños pequeños.

Ante esta situación se origina, por parte de las autoridades, cierta obligación de regular el auto cumplimiento de algunas normas éticas, así como de la obligación legal de actuaciones contra las empresas desleales contra el consumidor.

El objetivo de esta investigación es, concretamente, analizar y evaluar la publicidad de alimentos en dispositivos móviles dirigida a menores, antes y después de la aprobación del código, para, de este modo, a manera de objetivos o propósitos específicos de la investigación se busca:

1. Evaluar el seguimiento del código PAOS por parte de los anunciantes de alimentación en relación con las piezas publicitarias observadas en el periodo de estudio.

2. Derivado del anterior, valorar la existencia de una ética profesional en este sector publicitario.

3. Extraer un listado de los principales anunciantes de productos alimentarios que abordan a los menores de 12 años a través de apps, juegos interactivos o publicidad en formatos tradicionales.

4. Poner de manifiesto, mediante datos específicos, la influencia de la publicidad de productos infantiles alimentarios en la intención de compra de progenitores y cuidadores tras observar la publicidad.

${ }^{4}$ OCDE: Organización para la Cooperación y el Desarrollo Económico, integrada, en 2019, por: Australia, Austria, Bélgica, Canadá, Chile, Colombia, República Checa, Dinamarca, Estonia, Finlandia, Francia, Alemania, Grecia, Hungría, Islandia, Irlanda, Israel, Italia, Japón, Corea, Letonia, Luxemburgo, México, Holanda, Nueva Zelanda, Noruega, Polonia, Portugal, República de Eslovaquia, Eslovenia, España, Suecia, Suiza, Turquía, Reino Unido y Estados Unidos. 
Uso de smartphones en la infancia y seguimiento del Código PAOS por parte de anunciantes de alimentación

\section{METODOLOGÍA}

La metodología empleada es mixta, pues se acude a un análisis de contenido completada con una serie de entrevistas y un focus group.

En relación con el análisis de contenido, la muestra fue obtenida a través de la grabación durante 7 días (semana del 19 al 25 de agosto de 2019) de los contenidos publicitarios emitidos por los anunciantes, insertos en contenidos dirigidos a la población infantil.

Aunque actualmente existe un horario protegido en televisión (que comprende desde las 6:00 a las 22:00 horas), esto no es aplicable a la publicidad en dispositivos móviles, cuyo control parental ha de ser activado por los progenitores o cuidadores de los menores. Es, por esta razón, por la que las grabaciones tuvieron lugar en diferentes horas durante todo el día, comprendiendo las franjas entre las 10:00 y las 21:00 h.

El método de análisis se basó, por una parte, en un análisis de contenido de la publicidad de alimentos y bebidas dirigida a menores de 12 años en los dispositivos móviles en España. En concreto, analizamos la publicidad dirigida en menores a través de Musical.ly, Snapchat, YouTube, Instagram y BabyTV App. El criterio de elección de estas aplicaciones y páginas fue, básicamente, la audiencia (IAB Spain, 2019).

Con este análisis de contenido se pretendió, básicamente, detectar los principales anunciantes y marcas que utilizan con frecuencia la publicidad en estas aplicaciones, portales o redes sociales.

Los anuncios fueron clasificados conforme a su cumplimiento en referencia al Código PAOS y sus normas éticas en tres diferentes niveles: a) conforme; b) no conforme; y c) incierto (León et al., 2017). Haciendo uso de esta clasificación, y en relación con su visualización por parte de los investigadores, se tipificó la muestra y se tabularon dichos datos. Se evaluó el cumplimiento de cada norma considerando que estos cumplían enteramente con el Código PAOS cuando no se elude ninguno de los requisitos o estándares, es decir, fueron considerados no conformes si más del $50 \%$ de los estándares internos a cada norma fueron incumplidos. Aquellos cuyo cumplimiento fue carente del consenso, por parte de investigadores y expertos externos e imparciales, fueron considerados de cumplimiento incierto.

Por su parte, y previo consentimiento informado por parte de los padres, de acuerdo con los Códigos de Conducta para el tratamiento de datos de carácter personal en la Investigación de Mercados (2018) a nivel cuantitativo, se realizó una encuesta a un total de 524 niños de entre 5 y 12 años de edad y madres/padres de menores de las mismas edades. En concreto: 209 menores y 315 adultos, siendo estos progenitores 0 cuidadores. Ello se consiguió gracias a la herramienta Formularios de Google. Por otro lado, se obtuvieron datos de la aplicación de una metodología cualitativa basada en la realización de un focus group realizado a 8 madres y 2 padres.

Revista de Comunicación y Salud, 2020, Vol. 10, nº 3, pp. 53-72 
Uso de smartphones en la infancia y seguimiento del Código PAOS por parte de anunciantes de alimentación

\section{RESULTADOS}

\subsection{Resultados del análisis de contenido}

Durante los 7 días que tuvo lugar la recogida de muestras (11 horas al día), se contabilizó un total de 77 horas donde se encontraron un total de 314 anuncios dirigidos directamente al público infantil. De ellos, 73 fueron anuncios de comida o bebida.

De los 73 anuncios que componían la muestra total directamente relacionada con el objeto de estudio, 22 fueron los anunciantes totales. De este modo, las marcas responsables de esta publicidad fueron (por orden de aparición): 1) Ositos; 2) Babybel; 3) Fanta; 4) Danone; 5) Danonino; 6) La vaca que ríe; 7) Galletas Príncipe; 8) Chips Ahoy; 9) Choco Flakes; 10) Haribo; 11) Coca Cola sin cafeína; 12) Danup; 13) Telepizza; 14) Hero baby; 15) McDonalds; 16) Donuts; 17) Burger King; 18) Tosta Rica; 19) Puleva; 20) Oceanix; 21) Dinosaurus; y 22) Sunny Delight.

Evaluando el nivel de cumplimiento del código PAOS, y aunque lo representamos gráficamente en la siguiente tabla, el resumen es muy básico: Ninguna de estas marcas cumple lo establecido en el código PAOS al $100 \%$. Así lo vemos en la tabla 1:

Tabla 1.

Nivel de cumplimiento del Código PAOS

\begin{tabular}{|c|c|c|}
\hline № & Marca & Nivel de cumplimiento \\
\hline 1 & Ositos & No conforme \\
\hline 2 & Babybel & Incierto \\
\hline 3 & Fanta & No conforme \\
\hline 4 & Danone & Incierto \\
\hline 5 & Danonino & No conforme \\
\hline 6 & La vaca que ríe & Incierto \\
\hline 7 & Galletas Príncipe & No conforme \\
\hline 8 & Chips Ahoy & No conforme \\
\hline 9 & Choco Flakes & No conforme \\
\hline 10 & Haribo & No conforme \\
\hline 11 & $\begin{array}{l}\text { Coca Cola sin } \\
\text { cafeína }\end{array}$ & No conforme \\
\hline 12 & Danup & No conforme \\
\hline 13 & Telepizza & No conforme \\
\hline 14 & Hero baby & No conforme \\
\hline 15 & McDonalds & Incierto \\
\hline 16 & Donuts & No conforme \\
\hline 17 & Burger King & No conforme \\
\hline 18 & Tosta Rica & No conforme \\
\hline 19 & Puleva & Incierto \\
\hline 20 & Oceanix & No conforme \\
\hline 21 & Dinosaurus & No conforme \\
\hline 22 & Sunny Delight & No conforme \\
\hline
\end{tabular}

Fuente: Elaboración propia (2019)

Revista de Comunicación y Salud, 2020, Vol. 10, n 3, pp. 53-72 
Uso de smartphones en la infancia y seguimiento del Código PAOS por parte de anunciantes de alimentación

\subsection{Resultados de la encuesta}

Se administró una encuesta breve donde se registró el nivel de recuerdo y reconocimiento (Sánchez, 1999) de las marcas, productos y contenido de la publicidad, en aras de analizar la posible eficacia publicitaria. En este sentido, se les preguntó a los niños encuestados si habían entrado en alguna de las redes sociales, aplicaciones o portales referidos (Musical.ly, Snapchat, YouTube, Instagram y BabyTV App) en los últimos 7 días. Del total de 617 personas que respondieron la encuesta, 524 habían visto alguna de las aplicaciones marcadas y, consecuentemente, la publicidad inserta en ellas.

Respondieron la encuesta 209 menores de 12 años (previo consentimiento informado de los padres y cuidadores) y 315 adultos, todos progenitores.

De entre los datos más relevantes destacan:

- Los 209 menores y los 315 adultos reconocían perfectamente las marcas anunciantes cuando se les mostraba el envase o se les mencionaba la marca.

- Del total de encuestados, un 24,04\% recordaba la marca o el producto sin estímulo externo previo. En concreto, el reparto quedaba del siguiente modo:

- 73 de los 209 menores $(34,92 \%$ de los menores.

- 53 de los 315 adultos (17,09\% de los adultos).

- En relación con la intención de compra, el 100\% de los 209 menores manifestaron su deseo de comer o beber alguno de los alimentos o bebidas que aparecen en el anuncio mostrado; sin embargo, cuando la misma pregunta es respondida por los padres, el porcentaje desciende significativamente, dando datos como el de los 315 adultos, sólo $14(4,44 \%)$ no tenían intención de comprar o consumir ninguno de los productos mostrados. Esto puede ser lógico en la medida en que el nivel de formación académica es mucho más alto; sin embargo, lo que llama la atención de los datos es que, aparte de estos 14 encuestados, 198 (62,85\%) hicieron algunas de las categorías codificadas como "inciertas" como primera opción de compra, consumo o ingestión, frente a las codificadas como "no conformes".

Las marcas mejor valoradas por los padres son, en orden:

1. Puleva; 2. Danone; 3. Danonino; 4. Danup; 5. La vaca que ríe; 6. Minibabybel.

\subsection{Resultados del grupo de discusión}

En relación con la cantidad de tiempo que los niños pasan frente a los medios de comunicación, las ocho personas que estaban en la mesa admitieron que los niños pasan demasiado tiempo frente a las tecnologías; sin embargo, cuando se les pidió que cuantificaran cuánto tiempo consideraban apropiado que los niños estuvieran frente a un smartphone, las respuestas variaron considerablemente. Así, en comparación con "10 minutos al día", en el caso de R. O. J., madre de dos niñas, de 7 y 9 años, respectivamente, encontramos el "2 horas como máximo", que fue contestado por el

Revista de Comunicación y Salud, 2020, Vol. 10, no 3, pp. 53-72 
padre de dos menores, una niña de 5 y un niño de 6 años. Entre estas bandas, se dieron respuestas intermedias que apostaban más por una moderación "sin contar exactamente los minutos", y "siendo conscientes de que la exposición a los móviles está ahí y no podemos evitarla". O bien, respuestas como "no me llevo muy bien y no me gusta, pero un poco de tiempo para dejarme respirar no nos hace daño a ninguno de los dos".

En cualquier caso, establecemos uno de los puntos centrales de importancia de todas las respuestas en el hecho de que los padres, al referirse al tiempo que los menores están con un dispositivo, lo hacen en soledad, y no acompañados por un padre.

En este sentido, y refiriéndonos al siguiente bloque, todos los participantes en el foco eran conscientes de que los menores recibían impactos publicitarios, pero, en todos los casos, se pensaba que los impactos recibidos eran menores. Así, cuando se afirmó que durante una semana se recibió un promedio de más de 300 impactos, algunos de los comentarios inmediatos fueron en línea con "de ahora en adelante no les dejaré mi teléfono móvil" o "no tenía idea de tal cantidad". Pero, además de esto, sabiendo que 73 anuncios estaban influyendo directamente en la dieta de sus hijos, las reacciones fueron diversas: Así, 7 de las personas del foco entendieron que era normal y que no se trataba de alimentos especialmente dañinos para la salud, con alguna incidencia específica: "La Coca Cola no es muy buena... pero sin cafeína, beberla una vez a la semana no me parece muy malo". O "los yogures son muy buenos, siempre se ha dicho". La verdad es que sólo 3 de los participantes cuestionaron los ingredientes, las cantidades de azúcares, colorantes y conservantes, así como el concepto de alimentación saludable como base.

Como resultado final del enfoque, 4 de los participantes admitieron que compraron muchos productos alimenticios debido a las presiones e incitaciones de sus hijos, incluso cuando no estaban de acuerdo. E incluso en un caso:

Una vez dices que no, otra vez también. Pero cuando la galleta viene con La Patrulla Canina, y tus hijos la ven una y otra vez en la publicidad, a ver cómo les dices que no la compras para el desayuno... Además, la fruta ni siquiera quieren verla. Y si van a la escuela con el estómago vacío, o si van a la escuela con las galletas, jentonces La Patrulla Canina!.

En los 10 casos admitieron que muchos de los productos alimenticios que compraron fueron hechos directamente a través de la publicidad (ya sea la marca original o una marca blanca sustituta) o a petición expresa de sus hijos que, en muchas ocasiones, "por supuesto, lo ven en la publicidad y luego te preguntan". En este sentido, el objetivo cuarto queda alcanzado al acercarnos a la influencia de la publicidad de productos infantiles alimentarios en la intención de compra de los entrevistados tras observar la publicidad.

\section{CONCLUSIONES}

¿Cómo se evalúan actualmente los riesgos, las amenazas y las oportunidades de la Internet y las pantallas inteligentes utilizando diferentes modelos y enfoques teóricos? ¿Qué aspectos no incluidos en los diseños tradicionales de los medios educativos y comunicativos tendrían que incorporarse y que no están presentes actualmente?

Revista de Comunicación y Salud, 2020, Vol. 10, nº 3, pp. 53-72 
Uso de smartphones en la infancia y seguimiento del Código PAOS por parte de anunciantes de alimentación

¿Debería trabajarse más en la alimentación en el entorno escolar y familiar? ¿Debería prohibirse la publicidad de alimentos? ¿Funciona el Código PAOS?

El contenido de la publicidad de alimentos dirigida a los niños no solo vulnera e infringe lo establecido el código de la PAOS (con lo que se deriva la existencia de una dudosa ética profesional en este sector publicitario), sino que, además, también pasa desapercibido para el público destinatario (consumidores, menores, compradores, padres). De esta manera, los niveles de recordación y reconocimiento son muy elevados, provocando un aumento de la probabilidad de intención de compra y, en consecuencia, de consumo, a pesar de tratarse de productos alimenticios cuyos ingredientes y componentes están claramente identificados como nocivos o insalubres por la Organización Mundial de la Salud.

El alcance de esta investigación abarca a los ámbitos tanto económico como sanitario. Así, el hecho de quebrantar los estándares de los códigos puede conllevar un aumento de cuota de ventas en productos de alimentación infantil, al tiempo que afecta a la sanidad pública, en tanto en cuanto puede generar una predisposición física y psicológica que favorezcan el desarrollo de enfermedades y/o situaciones de fragilidad en la salud infantil, adecuando una base de predisposición a enfermedades como la obesidad.

Las implicaciones pueden plantearse en tres líneas: A nivel legal, supone la impunidad de la violación de un código legislativo enfocado y planteado, precisamente, para proteger a un colectivo de especial protección legal, en tanto en cuanto son influenciables: los menores; a nivel social, significa que un público especialmente débil y susceptible de convertirse en prescriptor, además de en consumidor, queda expuesto a merced de las estrategias y tácticas publicitarias sin una ética razonable; $y$, por último, a nivel ético, deja expuesto un sector económico, el de la alimentación, que siendo consciente de la infracción e incumplimiento del código, decide vulnerarlo para conseguir sus objetivos empresariales.

Los planteamientos proyectivos del estudio se enfocan en la visibilización de las causas y efectos de una situación que, siendo capaz de transformarse, debe, para ello, acometer evoluciones en varios frentes: el normativo, el social y el educacional, entre otros. En este sentido, cabe mencionar, como derivación de esta situación, la noticia aparecida en los medios en octubre de 2020 donde se indicaba que el Ministerio de Consumo del Gobierno de España reformará el Código PAOS prohibiendo la publicidad de alimentos no saludables que vaya dirigida a menores de 15 años (Molins, 2020). Esta novedad en la autorregulación pone de manifiesto las conclusiones a las que se han llegado en el Estudio Aladino 2019 (AESAN, 2020) donde se respaldan las ideas, con otros estudios, de las conclusiones y resultados aportados en este estudio.

En este sentido, y a la luz de los datos extraídos, podemos afirmar que, a pesar de la existencia de un código fuerte, dentro de una estrategia global (NAOS), su aplicación no está resultando efectiva, probablemente debido a la laxitud de su práctica, así como a la práctica inexistencia de sanciones que penalicen la violación de los principios 
Uso de smartphones en la infancia y seguimiento del Código PAOS por parte de anunciantes de alimentación

contemplados en el código del PAOS. Es por ello por lo que se hace necesario visibilizar con datos la situación y consecuencias de la no efectividad del actual código PAOS.

\section{REFERENCIAS}

Abramovitz, B. A. \& Birch, L. L. (2000). Five-year-old girls' ideas about dieting are predicted by mothers' dieting. Journal of the American Dietetic Association, 100(10), 1157-1163. doi: https://doi.org/10.1016/S0002-8223(00)00339-4

AESAN (2020). Estudio Aladino 2019. Estudio sobre la Alimentación, Actividad Física, Desarrollo Infantil y Obesidad en España. Ministerio de Consumo. Gobierno de España. Recuperado de https://www.aesan.gob.es/AECOSAN/web/nutricion/detalle/aladino 2019.htm

AIMC (2017). Estudio de penetración de medios convencionales y no convencionales. Asociación para la Investigación de Medios de Comunicación.

Bird, E. L., Halliwell, E., Diedrichs, Ph.C. \& Harcourt, D. (2013). Happy Being Me in the UK: A controlled evaluation of a school-based body image intervention with preadolescent children. Body Image, 10(3), 326-334. doi: https://doi.org/10.1016/j.bodyim.2013.02.008

Botta, R. (1999). Television images and adolescent girls, body image disturbance. En Journal Of Communication, 49 (2), 22-41. doi: https://doi.org/10.1111/i.14602466.1999.tb02791.x

Boyland, E. J., Harrold, J. A.; Kirkham, T. C. \& Halford, J. C. G. (2011): The extent of food advertising to children on UK television in 2008. International Journal of Pediatric Obesity, 6, 455-461. doi: https://doi.org/10.3109/17477166.2011.608801

Bringué, X. \& Sádaba, C. (2011). La generación interactiva en Madrid. Niños y adolescentes ante las pantallas. Madrid: Fundación Telefónica. Recuperado de https://hdl.handle.net/10171/17155

Carlson, L. \& Clarke, B. (2014). Reassessing the current state of advertising to children. International Journal of Advertising, 33(3), 429-36. doi: https://doi.org/10.2501/lJA-33-3-429-436

Carrillo, M. V., Jiménez Morales, M. \& Sánchez, M. (2011). Factores socioculturales y personales relacionados con el mensaje mediático del culto al cuerpo influyentes en el bienestar de los jóvenes. Comunicación y sociedad, 24(2), 227-252. Recuperado de https://revistas.unav.edu/index.php/communication-and-

society/article/view/36220/30737 
Uso de smartphones en la infancia y seguimiento del Código PAOS por parte de anunciantes de alimentación

Charry, K. M. (2014). Product placement and the promotion of healthy food to preadolescents: When popular TV series make carrots look cool. International Journal of Advertising 33(3), 599-616. doi: https://doi.org/10.2501/IJA-33-3-599-616

Códigos de Conducta para el tratamiento de datos de carácter personal en la Investigación de Mercados (2018). Recuperado de https://bit.ly/3eNuxgw

Código PAOS (2012). Código de co-regulación de la publicidad de alimentos y bebidas dirigidas a menores, prevención de la obesidad y la salud. Ministerio de sanidad, servicios sociales e igualdad. Gobierno de España. Recuperado de https://www.aesan.gob.es/AECOSAN/docs/documentos/nutricion/Nuevo Codigo PA OS 2012 espanol.pdf

Davison, K. K., Markey, C. N. \& Birch, L. L. (2003). A longitudinal examination of patterns in girls' weight concerns and body dissatisfaction from ages 5 to 9 years. International Journal Eating Disorders, 33, 320-332. doi: https://doi.org/10.1002/eat.10142

De Jans, S., Van de Sompel, D. Hudders, L. \& Cauberghe, V. (2019). Advertising targeting young children: an overview of 10 years of research (20062016). International Journal of Advertising, 38 (2), 173-206. doi: https://doi.org/10.1080/02650487.2017.1411056

Del Mar Pàmies, M., Ryan, G. \& Valverde, M. (2016). How intervention can empower children as consumers in dealing with advertising. International Journal of Consumer Studies, 40(5), 601-609. doi: https://doi.org/10.1111/ijcs.12305

Elías Zambrano, R., Jiménez-Marín, G. \& Silva Robles, C. (2017). Meios de comunicaçao audiovisual no contexto atual da Educaçao, en D. Caldevilla, Linguagens e Persuasao: Novas Criaçoes Narrativas (Ed., D. Caldevilla Domínguez). Ramada: Formalpress Publicaçoes e Marketing.

Fernández, E. \& Díaz Campo, J. (2014). La publicidad de alimentos en la televisión infantil en España: promoción de hábitos de vida saludables. Observatorio (OBS*), 8(4), 133150. Recuperado de http://obs.obercom.pt/index.php/obs/article/view/802

Folkvord, F., Anschütz, D. J., Boyland, E., Kelly, B. \& Buijzen, M. (2016). Food advertising and eating behavior in children. Current Opinion in Behavioral Sciences, 9, 26-31. doi: https://doi.org/10.1016/i.cobeha.2015.11.016

Frederick, D. A., Peplaua, L. A., \& Leverd, J. (2005). The swimsuit issue: Correlates of body image in a sample of 52,677 heterosexual adults. Body Image, 3(4), 413-419. doi: https://doi.org/10.1016/.j.bodyim.2006.08.002

Gerbner, G. (1998). Cultivation analysis: An overview. Mass Communication and Society, 3(4), 175-194. doi: https://doi.org/10.1080/15205436.1998.9677855

Revista de Comunicación y Salud, 2020, Vol. 10, n 3, pp. 53-72 
Uso de smartphones en la infancia y seguimiento del Código PAOS por parte de anunciantes de alimentación

González-Díaz, C. (2008). La categoría de producto y el mensaje transmitido en la publicidad infantil de alimentos. Revista Latina de Comunicación Social, 63, 480-491. doi: https://doi.org/10.4185/RLCS-63-2008-798-480-491

Gewerc, A., Fraga, F. \& Rodés, V. (2017). Niños y adolescentes frente a la Competencia Digital. Entre el teléfono móvil, youtubers y videojuegos. Revista Interuniversitaria de Formación del Profesorado, 89 (31.2), 171-186. Recuperado de http://hdl.handle.net/11162/166346

Haas, S. M., Irr, M. E., Jennings, N. A. \& Wagner, L. M. (2010). Communicating thin: A grounded model of Online Negative Enabling Support Groups in the pro-anorexia movement. New Media \& Society, 13(1), 40-57. doi: https://doi.org/10.1177/1461444810363910

Hamilton, K. \& Waller, G. (1993). Media influences on body size estimation in anorexia and bulimia. An experimental study. The British Journal of Psychiartry, 162(6), 837840. doi: https://doi.org/10.1192/bjp.162.6.837

Harrison, K. \& Marske, A. L. (2005): Nutritional Content of Foods Advertised During the Television Programs Children Watch Most. American Journal of Public Health, 95(9), 1568-1574. doi: https://doi.org/10.2105/ajph.2004.048058

Harrison, K. \& Cantor, J. (1997). The relationship between media consumption and eating disorders. Journal of Communication, 47(1), 40-67. doi: https://doi.org/10.1111/i.14602466.1997.tb02692.x

Hastings, G., Stead, M., McDermott, L., Forsyth, A. MacKintosh, A. M., Rayner, M., Godfrey, C., Caraher, M. \& Angus, K. (2003). Review Of Research On The Effects Of Food Promotion To Children. Food and Behaviour Research, 27, 51-63. Recuperado de https://www.fabresearch.org/viewltem.php?id=11053

Hendy, H. M., Gustitus, C. \& Leitzel-Schwalm, J. (2001). Social Cognitive Predictors of Body Image in Preschool Children. Sex Roles, 44(9), 551-569. doi: https://doi.org/10.1023/A:1012291008803

IAB Spain (2019). Estudio anual de Redes Sociales 2019. Interactive Advertising Bureau. Recuperado de https://iabspain.es/estudio/estudio-anual-de-redes-sociales-2019/

INFOADEX (2019). Estudio InfoAdex de la Inversión Publicitaria en España 2019. En InfoAdex. Recuperado de https://cutt.ly/OwH70jV

INTEF (2005). El poder de la publicidad. Instituto Nacional de Tecnologías Educativas y de Formación del Profesorado. Ministerio de Educación y Formación Profesional. Resuperado de http://cort.as/-QvBd 
Uso de smartphones en la infancia y seguimiento del Código PAOS por parte de anunciantes de alimentación

Jiménez-Morales, M. (2006). Cuando Barbarie se come a Garfield. Publicidad y alimentación: niños obesos buscando la perfección del cuerpo adulto. Trastornos de la Conducta Alimentaria, 3(2006), 245-263. Recuperado de http://www.tcasevilla.com/archivos/cuando barbie se come a garfield. publicidad y alimentacion.1.doc

Kapferer J. N. (1995). L'enfant et la publicité: les chemins de la seduction. Ed Dunod.

Kelly, B., Halford, J. C. G., Boyland, E. J., et al. (2010): Television Food Advertising to Children: A Global Perspective. doiAmerican Journal of Public Health, 100(9), 17301736. doi: https://doi.org/10.2105/ajph.2009.179267

Lara Martínez, M. \& Lara Martínez, A. (2018). Prejuicios y estereotipos en el cine sobre trastornos alimentarios. Revista de Comunicación y Salud, 8(2), 21-39. doi: https://doi.org/10.35669/revistadecomunicacionysalud.2018.8(2).21-39

León-Flández, K. A., Rico Gómez, A., Moya Geromin, M. Á., Romero Fernández, M., Bosqued Estefania, M. J., Damián, J., López Jurado, L. \& Royo Bordanada, M. Á. (2017). Evaluation of compliance with the Spanish Code of self-regulation of food and drinks advertising directed at children under the age of 12 years in Spain, 2012. Public Health, 150, 121-129. doi: https://doi.org/10.1016/j.puhe.2017.05.013

Levine, M. P. \& Murnen, S. K. (2009). Everybody knows that mass media are/are not [pick one] cause of eating disorders. A critical review of evidence for a causal link between media, negative body image and disordered eating in females. Journal of Social and Clinical Psychology, 28, 9-42. doi: https://doi.org/10.1521/jscp.2009.28.1.9

Ley 25/1994, de 12 de julio, por la que se incorpora al ordenamiento jurídico español la directiva europea sobre radiodifusión televisiva (TSF). Recpuerado de https://www.boe.es/eli/es///1994/07/12/25

Mancilla, A., Vázquez, R., Mancilla, J. M., Amaya, A. \& Álvarez, G. (2012). Body dissatisfaction in children and adolescents. A Systematic review. Revista Mexicana de Trastornos Alimentarios, 3(1), 62-69. Recuperdo de http://journals. iztacala.unam.mx/index.php/amta/article/view/212

Matthes, J. \& Naderer, B. (2015). Children's consumption behavior in response to food product placements in movies. Journal of Consumer Behaviour, 14(2), 127-36. doi: https://doi.org/10.1002/cb.1507

McCabe, M. P., Tatangelo, G., Watson, B., Fuller-Tyszkiewicz, M., Rodgers, R. F., Aimé, A., Mellor, D., Granero Gallegos, A., Strod, E., Caltabiano, M., Sicilia Camacho, A., CAstelnuovo, G., LoCoco, G., Grogan, S., Pobst, M., Dion, J., Maïano, C., Manzoni, G., Begin, C., Eve Blackburn, M., Pietrabissa, G., Markey, C., Gullo, S., Lirola, M. J., Alcaraz-lbáñez, M., Hayami-Chisuwa, N., He, Q., Ricciardelli, L. (2019). Development and testing of a model for risk and protective factors for eating disorders and higher

Revista de Comunicación y Salud, 2020, Vol. 10, n 3, pp. 53-72 
Uso de smartphones en la infancia y seguimiento del Código PAOS por parte de anunciantes de alimentación

weight among emerging adults: A study protocol. Body image, 31, 139-149. doi: https://doi.org/10.1016/j.bodyim.2019.10.001

McCabe, M. P. \& Ricciardelli, L. A. (2005). A longitudinal study of body image and strategies to lose weight and increase muscles among children. Applied Developmental Psychology, 26(5), 559-577. doi: https://doi.org/10.1016/j.appdev.2005.06.007

McLean, S., Paxton, S. J. \& Wertheim, E. H. (2013). Mediators of the relationship between media literacy and body dissatisfaction in early adolescent girls: Implications for prevention. Body Image, 10, 282-289. doi: https://doi.org/10.1016/j.bodyim.2013.01.009

Molins, A. (2020). Consumo prohibirá los anuncios de alimentos no saludables dirigidos a menores de 15 años. La Vanguardia, 9 de octubre de 2020. Disponible en https://bit.ly/2ltN8Ca

Myers, P. N. \& Biocca, M. (1992). The elastic body image: The effect of Television advertising and programming on body image distortion in Young women. Journal of Communication, 42(3), 103-133. doi: https://doi.org/10.1111/i.14602466.1992.tb00802.x

Navarro, H., González, Z., Massana, E., García, I. \& Contreras, R. (2012). El consumo multipantalla. Estudio sobre el uso de medios tradicionales y nuevos por parte de niños, jóvenes, adultos y personas mayores en Cataluña. Cuaderns del CAC 38, 15(1), 91-100. Recuperado de https://www.cac.cat/sites/default/files/2019$\underline{01 / \text { Q38 navarro et al ES.pdf }}$

Ortega, F. B., Cadenas-Sánchez, C., Sui, X., et al. (2015). Role of Fitness in the Metabolically Healthy but Obese Phenotype: A Review and Update. Progress in Cardiovascular Diseases, 58, 76-86. doi: https://doi.org/10.1016/j.pcad.2015.05.001

Pérez Tornero, J. M. (2008). La sociedad multipantallas: retos para la alfabetización mediática. Comunicar, 31, 15-25. doi: https://doi.org/10.3916/c31-2008-01-002

Phares, V., Steinberg, A. R. \& Thompson, K. (2004). Gender differences in peer and parental influences: Body image disturbance, self-worth, and psychological functioning in preadolescent children. Journal of Youth and Adolescence, 33, 421-429. doi: https://doi.org/10.1023/B:JOYO.0000037634.18749.20

Romero-Rodríguez, L. M., de-Casas-Moreno, P. \& Torres-Toukoumidis, Á. (2016). Dimensiones e indicadores de la calidad informativa en los medios digitales. Comunicar, 24(49), 91-100. doi: https://doi.org/10.3916/c49-2016-09

Sánchez Franco, M. (1999). Eficacia publicitaria: Teoría y práctica. Madrid: McGraw Hill. 
Uso de smartphones en la infancia y seguimiento del Código PAOS por parte de anunciantes de alimentación

Sánchez Muniz, F.J. (2016). Obesity: a very serious public health problem. Anales de la Real Academia Nacional de Farmacia, 82, pp. 6-26. Recuperado de https://analesranf.com/wp-content/uploads/2016/82 ex2/82ex2 02.pdf

Stice, E., Schupak-Neuberg, E., Shaw, H. E. \& Stein, R. I. (1994). Relation of media exposure to eating disorder symptomatology: An examination of mediating mechanisms. Journal of Abnormal Psychology, 103, 836-840. doi: https://doi.org/10.1037/0021-843X.103.4.836

Skemp-Arlt, K. M., Rees, K. S., Mikat, R. P. \& Seebach, E. E. (2006). Body mage dissatisfaction among third, fourth, and fifth grade children. Californian Journal of Health Promotion, 4(3), 58-67. doi: https://doi.org/10.32398/cjhp.v4i3.1958

Smith, R., Kelly, B., Yeatman, H. \& Boyland, E. (2019). Food Marketing Influences Children's Attitudes, Preferences and Consumption: A Systematic Critical Review. Nutrients, 11, p. 875.

Unnikrishnan, N., \& Bajpai, S. (1996). The Impact of TV Advertising on Children. New Delhi: Sage.

Vik, F. N., Bjørnarå, H. B., Øverby, N. C., Lien, N., Androutsos, O., Maes, L., Jan, N., Kovacs, E., Moreno, L. A., Dössegger, A., Manios, Y., Brug, J. \& Bere, E. (2013). Associations between eating meals, watching TV while eating meals and weight status among children, ages 10-12 years in eight European countries: the ENERGY crosssectional study. International Journal of Behavioral Nutrition and Physical Activity, 10(58), 1-10. doi: https://doi.org/10.1186/1479-5868-10-58

WHO - World Health Organization (2016). Informe de la comisión para acabar con la obesidad infantil. Recuperado de: https://cutt.ly/9r94HOE

\section{AUTORES}

\section{Gloria Jiménez-Marín}

Profesora en el área de Publicidad y RR.PP. en la Facultad de Comunicación de la Universidad de Sevilla y colaboradora en la UOC. Licenciada en Periodismo y Licenciada en Publicidad y RR.PP., es Máster en diseño publicitario. Tras su paso por la actividad profesional en agencias de publicidad y su labor docente en varias universidades españolas, su actividad investigadora se centra en la publicidad y las RR.PP., centrándose en cuestiones como el merchandising o los efectos de los medios. Cuenta con publicaciones en revistas como Revista Mediterránea de Comunicación, Revista Latina de Comunicación Social o Prisma Social. Es directora de la revista científica IROCAMM.

Orcid ID: $0000-0003-0252-3975$

Google Scholar: https://scholar.google.es/citations?user=pNLUjXUAAAAJ\&hl=es

ResearchID: E-5845-2010 


\section{Paloma Sanz-Marcos}

Profesora en el Dpto. de CAV y Publicidad de la Universidad de Sevilla. Doctora en Comunicación (mención internacional por la UC Berkeley, California) y Lda. en Publicidad y RR.PP., compagina sus labores docentes con la investigación en el área de la comunicación. Miembro del grupo de investigación IDECO, ha presentado comunicaciones en diversos encuentros académicos y ha publicado artículos y capítulos de libros sobre publicidad y brand management. Ha sido profesora visitante en universidades extranjeras como UC Berkeley, Universidade do Algarve (Portugal) o la Pontificia Universidad Católica de Chile.

Orcid ID: 0000-0002-6103-6993

Google Scholar: https://publons.com/researcher/1748618/paloma-sanz-marcos/

ResearchID: R-8935-2017

\section{Rodrigo Elías Zambrano}

Doctor en Comunicación es Licenciado en Comunicación Audiovisual y Máster en Gestión de Empresas AV por la Universidad de Sevilla, Máster en Comunicación y Educación AV por la Universidad de Huelva y Experto en E-Learning. A nivel docente, ha sido profesor en el Departamento de Marketing y Comunicación de la Universidad de Cádiz siendo, actualmente, Profesor Ayudante Dr. en el área de Publicidad y RR.PP. en la Facultad de Comunicación de la Universidad de Sevilla. Es, además, miembro del Grupo de investigación SEJ420. A nivel profesional, está ligado al mundo de la realización AV publicitaria para programas de producción propia y para productoras con servicio a Canal Sur, Tele5, Antena3 o, a nivel internacional, para TV I.N.S. (Instant news services) en Bruselas con servicio a la RAI, la CNBC, Al Jazeera, TVP o NHK.

Orcid ID: 0000-0001-8256-582X

GoogleScholar: https://scholar.google.es/citations?user=659XcUcAAAAJ\&hl=es

ResearchID: AAF-7865-2020 